\title{
FATORES ASSOCIADOS À INSEGURANÇA ALIMENTAR EM PESSOAS QUE VIVEM COM HIV/AIDS
}

\section{Factors associated with food insecurity among people living with HIV/AIDS \\ Factores asociados con la inseguridad alimentaria de personas con VIH /SIDA}

\author{
Lorena Nogueira Frota da Costa \\ Escola de Saúde Pública do Ceará - ESP - Fortaleza (CE) - Brasil \\ Universidade Estadual do Ceará - UECE - Fortaleza (CE) - Brasil \\ Hospital São José de Doenças Infecciosas do Ceará - HSJ - Fortaleza (CE) - Brasil
}

\section{Mayra Madeira Braga}

Escola de Saúde Pública do Ceará - ESP - Fortaleza (CE) - Brasil

Hospital São José de Doenças Infecciosas do Ceará - HSJ - Fortaleza (CE) - Brasil

\section{Marlene da Rocha}

Universidade Estadual do Ceará - UECE - Fortaleza (CE) - Brasil

\section{Mauricélia da Silveira Lima}

Escola de Saúde Pública do Ceará - ESP - Fortaleza (CE) - Brasil

Hospital São José de Doenças Infecciosas do Ceará - HSJ - Fortaleza (CE) - Brasil

\section{Wilma Félix Campêlo}

Hospital São José de Doenças Infecciosas do Ceará - HSJ - Fortaleza (CE) - Brasil

\section{Cláudia Machado Coelho Souza de Vasconcelos}

Hospital São José de Doenças Infecciosas do Ceará - HSJ - Fortaleza (CE) - Brasil

Universidade de Fortaleza - UNIFOR - Fortaleza (CE) - Brasil

\section{RESUMO}

Objetivo: Analisar fatores associados à insegurança alimentar em pessoas que vivem com HIV/AIDS. Métodos: Estudo transversal, realizado com 205 pacientes atendidos, de dezembro de 2016 a abril de 2017, em um hospital de referência em doenças infecciosas do estado do Ceará. Foram coletados dados socioeconômicos (renda, ocupação, condições de residência, escolaridade), demográficos (sexo, idade, estado civil), antropométricos (peso e altura), sobre hábitos (uso de álcool, drogas e práticas de atividade física) e de segurança alimentar. Avaliou-se a insegurança alimentar por meio da Escala Brasileira de Insegurança Alimentar, e as suas associações, através do teste qui-quadrado de Pearson e razão de prevalência. Resultados: Do total de 205 pacientes, 68,3\% (n=140) eram homens; 56,1\% ( $\mathrm{n}=115)$ tinham de 39 a 58 anos; $61 \%,(n=125)$ estavam solteiros; $60 \%(n=124)$ não estavam empregados; $49,8 \%(n=102)$ possuíam renda inferior a 1 e $1 \frac{1}{2}$ salário-mínimo; 38,5 $\%(\mathrm{n}=79)$ possuíam apenas nível fundamental; 47,3\% $(\mathrm{n}=97)$ estavam sobrepesados; 70,7\% ( $\mathrm{n}=145)$ moravam com 04 ou menos pessoas; $79,5 \%(\mathrm{n}=163)$ não consumiam álcool; 87,3\% $(\mathrm{n}=179)$ não usavam drogas e $64,4 \%(\mathrm{n}=132)$ não praticavam atividade física. A insegurança alimentar foi de 47,3\% (n=97). Desses, 10,7\% (n=22) estavam em nível grave. Encontrou-se associações estatísticas entre insegurança alimentar e faixa etária $(p=0,009)$, escolaridade $(p=0,013)$, número de pessoas por domicílio $(p<0,001)$ e renda mensal domiciliar $(p<$ 0,001). Conclusão: Observou-se alta prevalência de insegurança alimentar na amostra, que esteve associada às características demográficas (idade) e socioeconômicas (renda, escolaridade e condições de moradia).

Descritores: Segurança Alimentar e Nutricional; Vulnerabilidade Social; Síndrome de Imunodeficiência Adquirida.

\section{ABSTRACT}

Objective: To analyze factors associated with food insecurity in people living with HIV/AIDS. Methods: Cross-sectional study carried out with 205 patients treated, from December 2016 to April 2017, at a referral hospital in infectious diseases in the state of Ceará. The study collected socioeconomic (income, occupation, housing conditions, schooling), demographic (sex, age, marital status), and anthropometric (weight and height) data, along with data on habits (use of alcohol, drugs and practice of physical activity) and food safety. Food insecurity was evaluated through the Brazilian Food Insecurity Scale, and its associations, with use of Pearson's chi-square test and prevalence ratio. Results: Out of 205 patients, $68.3 \%(n=140)$ were men; $56.1 \%(n=115)$ were 39 to 58 years old; $61 \%(n=125)$ were single; $60 \%(n=124)$ 
were unemployed; $49.8 \%(n=102)$ had income lower than $11 \frac{1}{2}$ minimum wage; $38.5 \%(n=79)$ had studied only up to middle school; $47.3 \%$ $(n=97)$ were overweight; $70.7 \%(n=145)$ lived with 4 or fewer people; $79.5 \%(n=163)$ did not use alcohol; $87.3 \%(n=179)$ did not use drugs, and $64.4 \%(n=132)$ did not practice any physical activity. Food insecurity was $47.3 \%(n=97)$. Of these, $10.7 \%(n=22)$ were at a serious level. Statistical associations were found between food insecurity and age group ( $p=0.009)$, schooling ( $p=0.013)$, number of people per household $(p<0.001)$, and monthly household income $(p<0.001)$. Conclusion: A high prevalence of food insecurity was observed in the sample, and it was associated with demographic (age) and socioeconomic characteristics (income, schooling and housing conditions).

Descriptors: Food and Nutrition Security; Social Vulnerability; Acquired Immunodeficiency Syndrome.

\section{RESUMEN}

Objetivo: Analizar los factores asociados con la inseguridad alimentaria de personas con el VIH/AIDS. Métodos: Estudio transversal realizado con 205 pacientes asistidos entre diciembre de 2016 y abril de 2017 en un hospital de referencia en enfermedades infecciosas del estado de Ceará. Se recogieron los datos socioeconómicos (renta, ocupación, condiciones de vivienda, escolaridad), demográficos (sexo, edad, estado civil), antropométricos (peso y altura), sobre los hábitos (uso del alcohol, drogas y prácticas de actividad fisica) y de seguridad alimentaria. Se evaluó la inseguridad alimentaria a través de la Escala Brasileña de Inseguridad Alimentaria y sus asociaciones a través de la prueba Chi-cuadrado de Pearson y la razón de prevalencia. Resultados: Del total de 205 pacientes, el 68,3\% ( $n=140)$ eran hombres; el 56,1\% (n=115) tenían entre 39 y 58 años; el 61\%, $(n=125)$ eran solteros; el 60\% (n=124) sin trabajo; el 49,8\% ( $n=102)$ tenían renta inferior a 1 y 1/2 sueldo mínimo; el 38,5\% $(n=79)$ tenían solamente la educación primaria; el 47,3\% ( $n=97)$ tenían sobrepeso; el 70,7\% (n=145) vivian con 4 personas o menos; el 79,5\% $(n=163)$ no consumían el alcohol; el 87,3\% (n=179) no usaban drogas y el $64,4 \%$ (n=132) no practicaban actividad fisica. La inseguridad alimentaria ha sido del 47,3\% (n=97). De eses, el 10,7\% ( $n=22)$ estaban con el nivel grave. Se encontró asociaciones estadísticas entre la inseguridad alimentaria y la franja de edad ( $p=0,009)$, la escolaridad ( $p$ $=0,013)$, el número de personas por domicilio $(p<0,001)$ y la renta mensual del domicilio $(p<0,001)$. Conclusión: Se observó elevada prevalencia de inseguridad alimentaria en la muestra que estuvo asociada con las características demográficas (edad) y socioeconómicas (renta, escolaridad y condiciones de vivienda).

Descriptores: Seguridad Alimentaria y Nutricional; Vulnerabilidad Social; Síndrome de Inmunodeficiencia Adquirida.

\section{INTRODUÇÃO}

A Segurança Alimentar e Nutricional (SAN) parte do princípio de que todos têm o direito à alimentação regular e permanente, desde o acesso até a mesa. $\mathrm{O}$ alimento oferecido deve ser apropriado em quantidade suficiente e com qualidade, sem comprometer outras necessidades básicas, como educação, saúde e moradia. Além disso, a segurança alimentar deve também respeitar a dignidade e a diversidade cultural das pessoas. Ela é o direito de todos de se alimentarem com dignidade ${ }^{(1)}$.

Com o intuito de garantir uma alimentação saudável e adequada a todos, o Brasil faz uso de instrumentos legais que estão inseridos na Política Nacional de Segurança Alimentar e Nutricional (PNSAN), por meio da Lei Federal 11.346, de 15 de setembro de 2006, conhecida como Lei Orgânica de Segurança Alimentar e Nutricional (LOSAN) ${ }^{(1)}$, e da Emenda Constitucional n ${ }^{\circ} 64$ da Constituição de 1988 que, em 2010, incluiu a alimentação como um direito social, sendo considerada um marco na luta pelo direito humano à alimentação( ${ }^{(2)}$.

Ao contrário da SAN, a insegurança alimentar e nutricional (IAN) ocorre quando há violação desses direitos, impossibilitando acesso adequado, seguro e na quantidade suficiente a alimentos e nutrientes. Ela pode ser caracterizada desde o medo e preocupação de não conseguir prover o alimento na mesa diariamente, até a falta de distribuição adequada de alimentos para a família e a falta do próprio alimento ${ }^{(3,4)}$.

A IAN, no Brasil, foi avaliada pela Pesquisa Nacional por Amostra em Domicílios (PNAD), em 2013. Encontrou-se o quantitativo de 52 milhões de moradores $(25,8 \%)$ vivendo em insegurança alimentar. Desses, 12 milhões estavam vivendo na zona rural, correspondendo a $40 \%$ da população rural ( $\mathrm{n}=30$ milhões). Dentre todas as regiões do país, o Nordeste foi identificado como a região que teve o maior número de domicílios em insegurança alimentar, correspondendo a 44,2\% ${ }^{(5)}$.

A insegurança alimentar pode ser identificada em diversas circunstâncias, entre elas: situações de fome, desnutrição, pobreza, desigualdades sociais, incerteza da aquisição do alimento, preços abusivos, doenças e outras ${ }^{(2,3)}$.

Dentre os grupos vulneráveis à IAN, destacam-se pessoas vivendo com HIV/AIDS. A insegurança alimentar e o HIV/ AIDS se relacionam em um ciclo vicioso que pode causar a deficiência de macro e micronutrientes, e a redução da adesão ao tratamento, podendo interferir, inclusive, na transmissão do HIV. O estigma também gera um ambiente mais suscetível à insegurança alimentar, devido à diminuição do apoio social ${ }^{(6)}$.

O número de pessoas vivendo com HIV/AIDS (PVHA) em 2015, no mundo, esteve em 36,7 milhões, com 2,1 milhões de novos casos e 1,1 milhões de mortes decorrentes de infecções pelo HIV/AIDS. No Brasil, encontrou-se 830 mil casos e, no Ceará, até o junho de 2016, foram notificados 16.790 casos de HIV/AIDS, sendo a região metropolitana de Fortaleza o local de maior concentração de $\operatorname{casos}^{(7,8)}$. 
Considerada como uma das principais causas de morbi-mortalidade em todo mundo, a insegurança alimentar está intimamente associada à epidemia do HIV. Estima-se que aproximadamente 1 bilhão de pessoas no mundo carecem da disponibilidade de alimentos suficientes, mostrando que a prevalência de insegurança alimentar em PVHA é particularmente elevada $^{(7)}$.

Diante da importante relação existente entre insegurança alimentar e o HIV/AIDS, da possibilidade de interferência direta na adesão do paciente ao tratamento e na progressão da doença, bem como da ausência de trabalhos publicados no Ceará, este estudo teve como objetivo analisar os fatores associados à insegurança alimentar em pessoas que vivem com HIV/AIDS.

\section{MÉTODOS}

Trata-se de um estudo transversal, observacional e analítico, com abordagem quantitativa, realizado com pacientes com HIV/AIDS atendidos no ambulatório de um hospital de referência em doenças infecciosas do estado do Ceará, no período de coleta de dados compreendido entre dezembro de 2016 a abril de 2017.

Determinou-se a amostra por conveniência utilizando o cálculo de amostragem para populações finitas ${ }^{(9)}$, através da média mensal de pacientes atendidos $(n=989)$. Considerou-se como desfecho de interesse o percentual de $44 \%$ de insegurança alimentar, média dos estudos no Brasil, com 90\% de confiança dos dados e um erro aceitável de 5\%; obtendo-se, no total, 205 pacientes para a entrevista.

O convite para participação na presente pesquisa ocorreu no momento anterior ou posterior às consultas com o médico ou outro profissional de saúde, nos dias do ambulatório de HIV/AIDS, e, após a assinatura do Termo de Consentimento Livre e Esclarecido, iniciaram-se as entrevistas em sala reservada.

Durante a entrevista, aplicou-se um questionário com variáveis socioeconômicas (renda, ocupação, condições de residência, escolaridade) e demográficas (sexo, idade, estado civil), com perguntas sobre hábitos de uso de álcool ou drogas, e práticas de atividade física. A Escala Brasileira de Insegurança Alimentar (EBIA) ${ }^{(10)}$ foi utilizada e realizou-se a avaliação antropométrica, coletando-se informações sobre peso e altura, seguindo as orientações do Ministério da Saúde ${ }^{(11)}$.

Para classificar o nível de insegurança alimentar da população, empregou-se a EBIA, composta de 15 perguntas de sim ou não, para a qual cada resposta afirmativa tem o valor de 1 ponto. Para as famílias com menores de 18 anos, os pontos de corte foram: 0 indica segurança (SAN); 01-05 indica insegurança leve (IAL); 06-10, insegurança moderada; (IAM), 11-15, insegurança grave (IAG). Para as famílias sem menores de 18 anos, os pontos de corte foram: 0, SAN; 01-03, IAL; 04-05, IAM e 06-08, IAG $^{(10)}$.

Para a avaliação antropométrica, peso e altura foram aferidos utilizando uma balança digital com estadiômetro acoplado $\left(\right.$ Balmak $^{\circledR}$ ) modelo BK-200F ${ }^{(11)}$. Determinou-se o estado nutricional utilizando o Índice de Massa Corpórea (IMC) para adultos e idosos, considerando os parâmetros da $\mathrm{OMS}^{(12)}$ de forma adaptada. Decidiu-se classificar os adultos em magreza (IMC $<18,5$ $\mathrm{kg} / \mathrm{m}^{2}$ ), eutrofia (IMC de 18,5 a $24,9 \mathrm{~kg} / \mathrm{m}^{2}$ ) e excesso de peso (IMC $>24,9 \mathrm{~kg} / \mathrm{m}^{2}$ ); e os idosos em magreza $\left(\mathrm{IMC}<22 \mathrm{~kg} / \mathrm{m}^{2}\right.$ ), eutrofia (IMC de 22 a $27 \mathrm{~kg} / \mathrm{m}^{2}$ ) e excesso de peso (IMC $>27 \mathrm{~kg} / \mathrm{m}^{2}$ ).

Utilizou-se o programa GraphPad Prism (versão 5.0) para a análise dos dados, considerando um nível de significância estatística de 5\%. Primeiramente, realizou-se análise descritiva para medir a frequência das variáveis em estudo. Em seguida, as prevalências de IAN, segundo as diferentes categorias das variáveis analisadas, também foram calculadas.

Para investigar a associação entre as variáveis independentes e a prevalência de insegurança alimentar, executou-se o teste qui-quadrado de Pearson. Para medir a intensidade da associação, utilizou-se a razão de prevalência (RP) bruta com um intervalo de confiança de 95\% (IC95\%), considerando-se associações estatisticamente significantes aquelas que obtiveram valor de $\mathrm{p}<0,05$.

O presente estudo recebeu aprovação pelo Comitê de Ética e Pesquisa com Seres Humanos do hospital de referência em doenças infecciosas do Ceará, sob o Parecer nº 1.803.201.

\section{RESULTADOS}

Avaliaram-se 205 pessoas que vivem com HIV/AIDS atendidas em um hospital de referência em doenças infecciosas do Ceará, dos quais: $68,3 \%(\mathrm{n}=140)$ eram do sexo masculino e $31,7 \%(\mathrm{n}=65)$ do feminino; com maior prevalência $(56,1 \%, \mathrm{n}=115)$ da faixa etária de 39 a 58 anos e de solteiros $(61 \%, n=125)$. Além disso, $60 \%(n=124)$ estavam desempregados, ou recebiam auxílio-doença / aposentadoria; e 49,8\% (n=102) da população apresentou renda domiciliar menor que 1,5 salário-mínimo. Com relação à escolaridade, $38,5 \%(n=79)$ estudaram somente até o ensino fundamental (Tabela I).

No quesito avaliação nutricional: $6,8 \%(\mathrm{n}=14)$ foram diagnosticados com magreza, 45,8\% $(\mathrm{n}=94)$ com eutrofia e 47,3\% $(\mathrm{n}=97)$ com sobrepeso. Um percentual significativo das pessoas investigadas morava em domicílios com 04 ou menos moradores $(70,7 \%, \mathrm{n}=145)$, não usava álcool $(79,5 \%, \mathrm{n}=163)$ nem drogas $(87,3 \%, \mathrm{n}=179)$ e não praticavam atividade física $(64,4 \%$, $\mathrm{n}=132$ ) (Tabela I). 
As associações entre insegurança alimentar e as variáveis encontram-se na Tabela I. Elas se apresentaram estatisticamente significantes entre a insegurança alimentar, faixa etária $(p=0,009)$, escolaridade $(p=0,013)$, número de pessoas por domicílio $(\mathrm{p}<0,001)$ e renda mensal domiciliar $(\mathrm{p}<0,001)$.

De acordo com as razões de prevalência e seus respectivos intervalos de confiança, observou-se que, para pessoas que vivem com HIV/AIDS, os indivíduos com menos de 59 anos $(\mathrm{RP}=1,98, \mathrm{IC}=1,07-3,66)$ e que estudaram somente até o ensino fundamental $(\mathrm{RP}=1,44, \mathrm{IC}=1,08$ - 1,91) e aqueles que contam com menos de 1,5 salário mínimo $(\mathrm{RP}=2,76, \mathrm{IC}=1,93-3,94)$ como renda familiar mensal têm maior probabilidade de estar em insegurança alimentar. No entanto, os domicílios que têm mais que 4 pessoas morando apresentam menor probabilidade (Tabela I).

Tabela I - Distribuição da segurança/insegurança alimentar, razão de prevalência (RP) bruta e intervalo de confiança (IC) em pessoas que vivem com HIV/AIDS atendidas em um hospital de referência em doenças infecciosas. Fortaleza, Ceará, 2017.

\begin{tabular}{|c|c|c|c|c|c|c|c|c|c|}
\hline \multirow[t]{2}{*}{ Variável } & \multicolumn{2}{|c|}{$\begin{array}{c}\text { Total de } \\
\text { Indivíduos } \\
(205) \\
\end{array}$} & \multicolumn{2}{|c|}{$\begin{array}{c}\text { Segurança } \\
\text { Alimentar } \\
\text { (108) }\end{array}$} & \multicolumn{2}{|c|}{$\begin{array}{c}\text { Insegurança } \\
\text { Alimentar } \\
(97) \\
\end{array}$} & \multirow[t]{2}{*}{$\underset{\text { Bruta }}{\text { RP }}$} & \multirow[t]{2}{*}{ IC 95\% } & \multirow[t]{2}{*}{$\begin{array}{l}\text { Valor } \\
\text { de } p^{*}\end{array}$} \\
\hline & $\mathrm{n}$ & $\%$ & n & $\%$ & $\mathbf{n}$ & $\%$ & & & \\
\hline Sexo & & & & & & & & & 0,060 \\
\hline Feminino & 65 & 31,7 & 28 & 43,1 & 37 & 56,9 & 1,33 & $0,99-1,77$ & \\
\hline Masculino & 140 & 68,3 & 80 & 57,1 & 60 & 42,9 & 1,00 & - & \\
\hline Faixa etária (anos) & & & & & & & & & 0,009 \\
\hline$<59$ & 174 & 84,9 & 85 & 48,8 & 89 & 51,2 & 1,98 & $1,07-3,66$ & \\
\hline$\geq 59$ & 31 & 15,1 & 23 & 74,2 & 8 & 25,8 & 1,00 & & \\
\hline \multicolumn{10}{|l|}{ Estado Civil } \\
\hline Solteiro & 125 & 61 & 66 & 52,8 & 59 & 47,2 & 1,03 & $0,72-1,47$ & 0,872 \\
\hline Casado & 48 & 23,4 & 26 & 54,2 & 22 & 45,8 & 1,00 & - & \\
\hline Divorciado & 13 & 6,3 & 6 & 46,2 & 7 & 53,8 & 1,17 & $0,65-2,12$ & 0,608 \\
\hline Viúvo & 19 & 9,3 & 10 & 52,6 & 9 & 47,4 & 1,03 & $0,59-1,82$ & 0,910 \\
\hline Escolaridade & & & & & & & & & 0,013 \\
\hline Até ensino fundamental & 79 & 38,5 & 33 & 41,8 & 46 & 58,2 & 1,44 & $1,08-1,91$ & \\
\hline Ensino médio e superior & 126 & 61,5 & 75 & 59,5 & 51 & 40,5 & 1,00 & - & \\
\hline \multicolumn{10}{|l|}{ Ocupação } \\
\hline Empregado & 81 & 39,5 & 41 & 50,6 & 40 & 49,4 & 1,00 & - & \\
\hline Desempregado & 58 & 28,3 & 24 & 41,4 & 34 & 58,6 & 1,19 & $0,87-1,62$ & 0,282 \\
\hline Auxílio doença ou aposentadoria & 66 & 32,2 & 43 & 65,2 & 23 & 34,8 & 0,71 & $0,48-1,05$ & 0,077 \\
\hline Casa Própria & & & & & & & & & 0,153 \\
\hline Sim & 139 & 67,8 & 78 & 56,1 & 61 & 43,9 & 1,00 & - & \\
\hline Não & 66 & 32,2 & 30 & 45,5 & 36 & 54,5 & 0,85 & $0,60-1,07$ & \\
\hline Número de pessoas por domicílio & & & & & & & & & $<0,001$ \\
\hline$\leq 4$ & 145 & 70,7 & 62 & 42,8 & 83 & 57,2 & 2,45 & $1,52-3,96$ & \\
\hline$>4$ & 60 & 29,3 & 46 & 76,7 & 14 & 23,3 & 1,00 & - & \\
\hline Renda mensal domiciliar & & & & & & & & & $<0,001$ \\
\hline$<1 \frac{1}{1} 2$ salário mínimo & 102 & 49,8 & 31 & 30,4 & 71 & 69,6 & 2,76 & $1,93-3,94$ & \\
\hline$\geq 1 \frac{1}{2} 2$ salário mínimo & 103 & 50,2 & 77 & 74,8 & 26 & 25,2 & 1,00 & - & \\
\hline Usōo de álcool & & & & & & & & & 0,091 \\
\hline $\operatorname{Sim}$ & 42 & 20,5 & 27 & 64,3 & 15 & 35,7 & 0,71 & $0,46-1,1$ & \\
\hline Não & 163 & 79,5 & 81 & 49,7 & 82 & 50,3 & 1,00 & - & \\
\hline Uso de drogas & & & & & & & & & 0,333 \\
\hline Sim & 26 & 12,7 & 16 & 61,5 & 10 & 38,5 & 0,79 & $0,48-1,32$ & \\
\hline Não & 179 & 87,3 & 92 & 51,4 & 87 & 48,6 & 1,00 & - & \\
\hline Prática de atividade Física & & & & & & & & & 0,105 \\
\hline Sim & 73 & 35,6 & 44 & 60,3 & 29 & 39,7 & 0,77 & $0,56-1,07$ & \\
\hline Não & 132 & 64,4 & 64 & 48,9 & 68 & 51,5 & 1,00 & - & \\
\hline \multicolumn{10}{|l|}{ Diagnóstico Nutricional (IMC**) } \\
\hline Magreza & 14 & 6,8 & 8 & 57,1 & 6 & 42,9 & 0,84 & $0,44-1,59$ & 0,567 \\
\hline Eutrofia & 94 & 45,8 & 46 & 48,9 & 48 & 51,1 & 1,00 & - & \\
\hline Excesso de peso & 97 & 47,3 & 54 & 55,7 & 43 & 44,3 & 0,87 & $0,64-1,17$ & 0,352 \\
\hline
\end{tabular}

* Teste de qui-quadrado de Pearson. ** IMC - Índice de Massa Corporal. 
Encontrou-se prevalência de insegurança alimentar de 47,3\% ( $\mathrm{n}=97)$, sendo: 24,9\% ( $\mathrm{n}=51)$ de insegurança leve (IAL), $11,7 \%(n=24)$ de insegurança moderada (IAM) e 10,7\% $(n=22)$ de insegurança grave (IAG). Apresenta-se o exposto na Tabela II.

Nos domicílios com insegurança alimentar, em 36,1\% $(\mathrm{n}=35)$ deles havia menores de 18 anos. Ao comparar os domicílios com e sem menores de 18 anos, a prevalência de IAG foi maior no primeiro grupo $(25,7 \%, \mathrm{n}=9)$ (Figura 1).

Tabela II - Distribuição de frequência de segurança e insegurança alimentar de pessoas que vivem com HIV/AIDS atendidas em um hospital de referência em doenças infecciosas, segundo a Escala Brasileira de Insegurança Alimentar (EBIA). Fortaleza, Ceará, 2017.

\begin{tabular}{lcc}
\hline EBIA & n & Frequência (\%) \\
\hline Segurança alimentar & 108 & 52,7 \\
Insegurança alimentar & 97 & 47,3 \\
Leve & 51 & 24,9 \\
Moderada & 24 & 11,7 \\
Grave & 22 & 10,7 \\
\hline
\end{tabular}

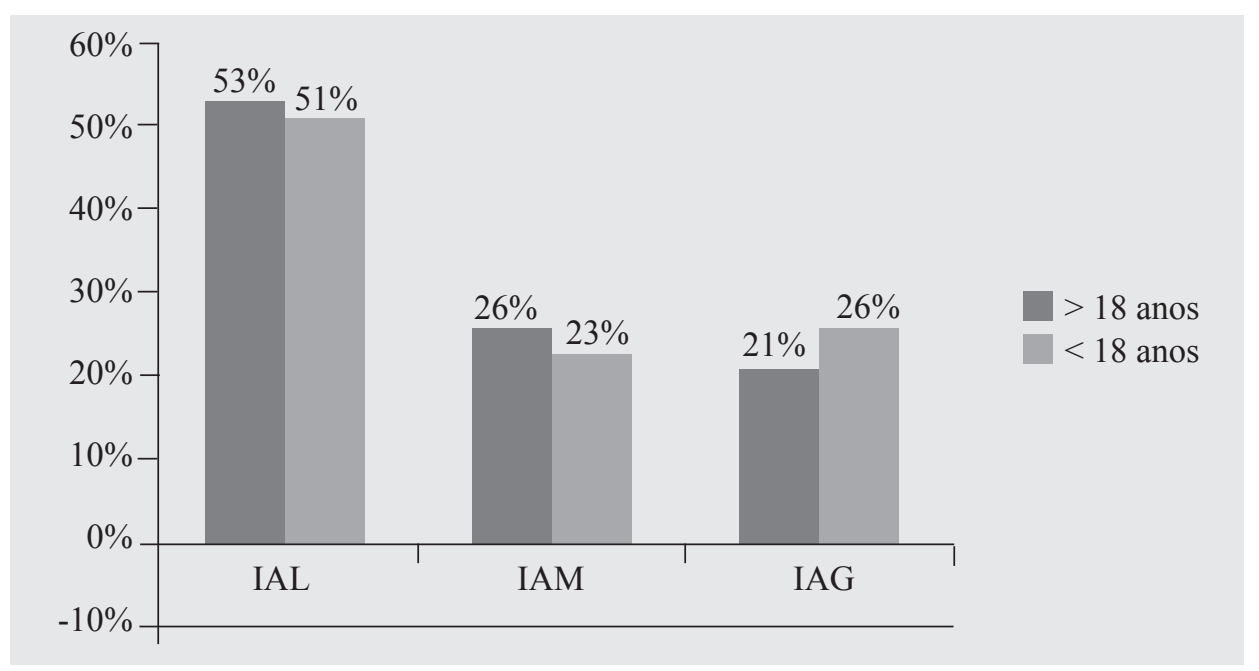

Figura 1 - Prevalência das diferentes condições de insegurança alimentar, segundo a existência ou não de menores de 18 anos nos domicílios de pessoas que vivem com HIV/AIDS atendidas em um hospital de referência em doenças infecciosas. Fortaleza, Ceará, 2017.

Legenda: IAL: Insegurança Alimentar Leve; IAM: Insegurança Alimentar Moderada; IAG: Insegurança Alimentar Grave.

\section{DISCUSSÃO}

No Brasil, em 2015, a maior concentração de casos de HIV/AIDS estava na faixa etária de 25 a 39 anos, com a maioria dos indivíduos pertencentes ao sexo masculino e com maiores ocorrências entre aqueles com formação escolar de $5^{\mathrm{a}}$ à $8^{\mathrm{a}}$ série incompleta $(29,8 \%)^{(7,13)}$. Quando o destaque é o Ceará, as características da população mudam um pouco, com taxas mais elevadas da doença na faixa etária de 30 a 39 anos, mas também concentradas no sexo masculino ${ }^{(8)}$.

A maior prevalência de pessoas do sexo masculino com HIV/AIDS ocorre no Brasil, especialmente no Ceará, como foi observado também nesta pesquisa. Esse fato já era esperado em decorrência da gradual redução dos casos entre mulheres e aumento entre homens. Informações evidenciadas pelo boletim epidemiológico, realizado em 2006, mostrou para cada 1 caso em mulher, havia 1,2 casos em homem. Esse valor aumentou em 2015, quando para cada 1 caso em mulher, havia 3 casos em homens ${ }^{(7,13)}$.

Em relação à faixa etária, observou-se que, no Ceará, ela é mais alta do que no restante do Brasil. Contudo, as encontradas no presente estudo são mais altas até mesmo do que a faixa etária do estado, pois está entre 39 e 58 anos, resultado acima do esperado. Esse fato pode ser devido à distribuição do HIV/AIDS não ocorrer de forma homogênea ${ }^{(14)}$.

Para a escolaridade, o atual estudo encontrou: $38,5 \%$ dos avaliados estudaram até o ensino fundamental e os demais $(61,5 \%)$ chegaram até o ensino médio ou superior. Trata-se de uma contradição com os dados do Brasil, que mostram a maior parcela dessa população estudando somente até o fundamental incompleto ${ }^{(13)}$. Esse dado foi considerado favorável para população de estudo, pois a baixa escolaridade é um preditor para a insegurança alimentar e reflete, mais uma vez, a distribuição não homogênea do HIV/AIDS ${ }^{(14)}$. 
Estudos realizados no Brasil com pacientes que vivem com HIV/AIDS também caracterizaram a população estudada, coincidindo com alguns resultados encontrados na presente pesquisa. Por exemplo, a prevalência da maioria masculina e da faixa etária mais alta do que a nacional (que está entre 43 anos e 45 anos), o que pode estar relacionado a uma mudança no perfil demográfico dessa população, com um aumento na faixa etária prevalente ${ }^{(15,16)}$. Com relação ao estado civil, somente um dos estudos brasileiros ${ }^{(16)}$ investigou esse quesito, e também encontrou um número significativo de pessoas solteiras. Talvez esse resultado esteja relacionado ao fato da doença ser sexualmente transmissível. No entanto, não se encontrou estudo que avaliasse a dificuldade de pacientes com HIV estabelecerem relacionamentos. Estudos futuros poderão verificar se existe esse tipo de dificuldade para esses pacientes.

Os estudos que associam insegurança alimentar e HIV/AIDS no Brasil são escassos, apesar da população nacional com HIV/AIDS e dos dados de insegurança alimentar serem grandes. Foram encontradas pesquisas somente em duas cidades, Brasília $^{(16)}$, Distrito Federal, e Limeira, São Paulo ${ }^{(15)}$, destacando seus principais achados para a associação da IAN com a baixa renda e o menor consumo de frutas e carnes ${ }^{(15)}$, além de baixo nível educacional e classe social ${ }^{(16)}$.

O HIV/AIDS está intimamente ligado à insegurança alimentar e ao estado nutricional, tendo em vista que essa associação leva à redução da adesão e da resposta à terapia anti-retroviral, reduzindo as células de diferenciação (CD4) e aumentando a carga viral. Tudo isso, juntamente com as infecções oportunistas, pode acentuar os impactos socioeconômicos na vida das pessoas que vivem com HIV/AIDS (PVHA), acelerando a progressão da doença e diminuindo os índices de sobrevivência individual ${ }^{(16,17)}$.

A ameaça que o HIV/AIDS exerce sobre a segurança alimentar foi reconhecida pela primeira vez somente no final dos anos 80 e início dos anos 90, apresentando na escolaridade e na renda familiar baixas alguns preditores para a insegurança alimentar(17).

Os dados do presente estudo para insegurança alimentar mostraram um elevado percentual (47,3\%) para a amostra avaliada. A IAG foi bastante elevada (10,7\%) quando comparada com os dados com a Pesquisa Nacional por Amostra em Domicílios (PNAD), a qual avaliou que, até 2013, havia um percentual de $25,8 \%$ de pessoas no Brasil que estavam vivendo em insegurança alimentar, das quais: $17,1 \%$ estavam em IAL, 5,1\% em IAM e 3,6 \% em IAG ${ }^{(5)}$.

Os resultados encontrados no atual estudo são maiores que os apresentados em nível de Brasil, mas, em comparação com a região Nordeste, os valores se aproximam. Vale destacar que essa região é identificada com o maior número de pessoas em insegurança alimentar do Brasil, correspondendo a $45 \%$ da população brasileira e 38,1\% da população do Nordeste, cujos valores encontrados foram: $23,6 \%$ de IAL, $8,9 \%$ de IAM e 5,6\% de $\operatorname{IAG}^{(5)}$. Houve exceção apenas para a IAG, que teve o percentual de quase o dobro do esperado. Isto pode ser devido à própria infecção pelo vírus, que pode causar debilidade dos membros da família e diminuir a capacidade econômica individual e familiar, o que acaba intensificando a insegurança alimentar dessa população( ${ }^{(6)}$.

Os estudos de insegurança alimentar em PVHA, no Brasil, também encontraram valores elevados para IAN quando comparados à prevalência nacional, apesar de não terem sido realizados no Nordeste. São exemplos os dados observados no Sudeste, em Limeira, São Paulo, onde a prevalência foi de $51 \%$ de IAN ${ }^{(15)}$, e no Centro-Oeste, em Brasília, Distrito Federal, com $36,8 \%$ de $\operatorname{IAN}^{(16)}$.

Em outros países, existem mais estudos sobre a relação do HIV/AIDS e a insegurança alimentar, como os achados na Etiópia $(63 \%)^{(17)}$, São Francisco $(54,6 \%)^{(18)}$, Columbia Britânica $(71,3 \%)^{(19)}$ e República Democrática do Congo $(57 \%)^{(20)}$, mostrando que a insegurança alimentar está presente em todos os locais, principalmente em populações marginalizadas e estigmatizadas.

Este estudo mostra também que havia menores de 18 anos em 36,1\% dos domić́lios avaliados com insegurança alimentar. Esse é um fator que pode agravar a situação de insegurança da família, pois se observou que a IAG é maior no grupo com menores de 18 anos, o que pode ter ocorrido porque os adultos do domicílio diminuíram a quantidade da sua alimentação para prover alimento em quantidade adequada para as crianças, fato também encontrado em uma pesquisa com crianças em Colombo (Paraná) ${ }^{(21)}$.

A insegurança alimentar em PVHA está, muitas vezes, relacionada ao estado nutricional do paciente, pois ele apresenta risco elevado para desnutrição devido à redução da ingestão de energia em decorrência da anorexia e da saciedade precoce. Igualmente, a IAN relaciona-se também aos efeitos sistémicos das co-infecções; da deglutição prejudicada por infecções oportunistas orais; e da incapacidade progressiva, o que leva ao desemprego e, consequentemente, à dificuldade em aquidirir alimentos. Além disso, a própria infecção pelo vírus aumenta as necessidades metabólicas e está associada à má absorção de gordura e carboidratos, o que piora a relação entre a desnutrição e a progressão da doençç ${ }^{(6)}$.

Ademais, a desnutrição por micronutrientes também é muito presente nas PVHA por meio do aumento do estresse oxidativo, o que pode elevar as cargas virais do HIV e, assim, aumentar a infectividade do indivíduo e a probabilidade de transmissão do HIV para outros ${ }^{(6)}$.

Apesar de as evidências relacionando a desnutrição em PVHA à insegurança alimentar serem fortes, o presente estudo não encontrou associação estatisticamente significante entre essas duas variáveis, o que provavelmente ocorreu devido ao percentual de magreza $(6,8 \%)$ da amostra estudada ter sido pequeno. 
Não só a desnutrição pode estar envolvida com a insegurança alimentar, mas também outros fatores, como a dependência da bebida alcoólica, o uso de drogas, a baixa renda e a pouca escolaridade ${ }^{(4)}$. Esses aspectos constituem o processo de incerteza do acesso a alimentos seguros, de forma socialmente aceitável, o que leva a experiências de fome ${ }^{(17)}$. A baixa escolaridade e a baixa renda familiar são características marcantes nos estudos de insegurança alimentar e HIV/AIDS, evidenciando que há associação entre essas variáveis. Os resultados desta e de outras pesquisas ${ }^{(15,16)}$ consolidam essa relação.

Outra associação encontrada neste estudo foi: para as pessoas com menos de 59 anos, a prevalência de IAN ;e o número de pessoas vivendo no mesmo domicílio revelou maior IAN (57,2\%) no grupo com 04 ou menos pessoas residindo juntas, resultado contrário ao esperado quando comparado com o PNAD, que trouxe um percentual de 8,2\% para domicílios com 04 a 06 moradores e de 6,5\% para os com até 03 moradores $^{(5)}$.

Até pouco tempo, a insegurança alimentar não podia ser mensurada, pois não existia instrumento oficial brasileiro que tivesse essa finalidade. Isto mudou quando uma ferramenta chamada de Escala Brasileira de Insegurança Alimentar (EBIA), adaptada da escala desenvolvida pelo United States Department of Agriculture (USDA) ${ }^{(10)}$, foi validada. A partir de então, essa escala vem sendo reconhecida como indicador sensível para identificar famílias vulneráveis à insegurança alimentar ${ }^{(22,23)}$.

A escala mensura a insegurança alimentar e a classifica em leve, moderada e grave, e ainda atesta a segurança alimentar do indivíduo caso haja a satisfação completa das necessidades alimentares. A EBIA está baseada nas condições econômicas e alimentares da família, nas percepções e experiências relatadas pelos indivíduos envolvidos ${ }^{(10,23)}$.

Todos os trabalhos analisados utilizaram a EBIA ou a USDA para mensurar a IAN através de estudos que seguiram caminhos metodológicos semelhantes, o que permite comparar os resultados encontrados. Além disso, a EBIA oferece várias vantagens na sua aplicação, como: aprender como as pessoas inseridas na insegurança alimentar e/ou na fome a percebem; captar as dimensões psicológicas e físicas das pessoas envolvidas; classificar os indivíduos e famílias de acordo com o nível de insegurança alimentar que estão vivendo, além do baixo custo da aplicação do instrumento ${ }^{(22,23)}$.

Para o controle da insegurança alimentar, o Brasil conta com a Lei Orgânica de Segurança Alimentar e Nutricional LOSAN, que visa garantir o direito humano à alimentação e considera que a segurança alimentar e nutricional abrange: acesso aos alimentos, sustentabilidade, promoção da saúde, qualidade biológica, sanitária, nutricional e tecnológica dos alimentos; diversidade cultural; acesso à informação e implementação de políticas públicas ${ }^{(1)} \mathrm{e}$, assim, na saúde coletiva.

Apesar de todos os artifícios implementados no Brasil para combater a insegurança alimentar, ela ainda é muito prevalente ${ }^{(5)}$, principalmente em populações marginalizadas e estigmatizadas com as PVHA. A escassez de estudos na área aumenta o desconhecimento e as incertezas sobre o assunto, o que torna relevantes os resultados desta pesquisa.

$\mathrm{O}$ atual estudo apresenta limitações por ser do tipo transversal, impossibilitando estabelecer relações causais entre as variáveis estudadas e o desfecho, além da amostragem ter sido aleatória, limitando os resultados para populações com características semelhantes. Destaca-se, entretanto, que os resultados podem revelar hipóteses interessantes para que outros estudos formulem as próprias hipóteses para análise de causalidade.

Diante da alta prevalência de insegurança alimentar encontrada para as pessoas que vivem com HIV/AIDS, compreende-se que essa pode interferir diretamente na adesão do paciente ao tratamento e na progressão da doença. O resultado desta pesquisa deve ser visto com cuidado, no intuito de que sejam realizadas mais intervenções de políticas públicas de saúde para garantir a segurança alimentar e nutricional dessa população.

\section{CONCLUSÃO}

Observou-se alta prevalência de insegurança alimentar na amostra, que esteve associada a características demográficas (idade) e socioeconômicas (renda, escolaridade e condições de moradia).

\section{REFERÊNCIAS}

1. Brasil. Lei n. ${ }^{\circ} 11.346$, de 15 de setembro de 2006. Cria o Sistema Nacional de Segurança Alimentar e Nutricional - SISAN com vistas em assegurar o direito humano à alimentação adequada e dá outras providências [acesso em 21 Maio 2017]. Disponível em: http://www.planalto.gov.br/ccivil_03/_ato2004-2006/2006/lei/111346.htm

2. Brasil. Constituição (1988). Emenda Constitucional n. ${ }^{\circ} 64$, de 4 de fevereiro de 2010. Altera o art. $6^{\circ}$ da Constituição Federal, para introduzir a alimentação como direito social . Diário Oficial da União; Brasília 04 fev 2010; Secção 1 [acesso em 21 Maio 2017]. Disponível em: http://www.planalto.gov.br/ccivil_03/constituicao/emendas/emc/emc64.htm

3. Santos JV. Prevalência de insegurança alimentar em famílias de Pelotas, RS e estado nutricional das pessoas que vivem em insegurança alimentar [dissertação]. Pelotas: Universidade Federal de Pelotas; 2007.

4. Silva CCS, Oliveira KBB, Alves AS, Neves JA, Modesto CAC, Vianna RPT. Association between consumption and food (in)security in food and nutrition of São José dos Ramos - PB. Braz J Food Technol. 2012; 15(Nesp):23-30. 
5. Instituto Brasileiro de Geografia e Estatística. Pesquisa Nacional por Amostra de Domicílios - PNAD 2013. Rio de Janeiro: IBGE; 2014.

6. Weiser SD, Young SL, Cohen CR, Kushel MB, Tsai AC, Tien PC, et al. Conceptual framework for understanding the bidirectional links between food insecurity and HIV/AIDS1234. Am J Clin Nutr. 2011;94(6):1729S-39S.

7. Joint United Nations Program on HIV/AIDS (UNAIDS). Get on the Fast-Track: the life-cycle approach to HIV. Geneva: UNAIDS; 2017.

8. Secretaria da Saúde do Estado do Ceará (SESA-CE). Boletim Epidemiológico HIV/AIDS 2016. Fortaleza: SESA/CE; 2016.

9. Santos GEO. Cálculo amostral: calculadora on-line [acessado 2017 Maio 16]. Disponível em: http://www.calculoamostral. vai.la

10. Segall-Corrêa AM, Marin-Leon L. A segurança alimentar no brasil: proposição e usos da Escala Brasileira de Medida da Insegurança Alimentar (EBIA) de 2003 a 2009. Segur Aliment Nutr. 2009;16(2):1-19.

11. Ministério da Saúde (BR), Secretaria de Atenção à Saúde, Departamento de Atenção Básica. Orientações para a coleta e análise de dados antropométricos em serviços de saúde: Norma Técnica do Sistema de Vigilância Alimentar e Nutricional SISVAN. Brasília: Ministério da Saúde; 2011 [acesso em 2017 Maio 21]. Disponível em: http://189.28.128.100/dab/docs/ portaldab/publicacoes/orientacoes_coleta_analise_dados_antropometricos.pdf

12. World Health Organization. Obesity: preventing and managing the global epidemic. Geneva: WHO; 1998.

13. Ministério da Saúde (MS). Secretaria de Vigilância em Saúde. Boletim Epidemiológico HIV/AIDS 2016. Brasília: Ministério da Saúde; 2016.

14. Brito AM, Castilho EA, Szwarcwald CL. AIDS e infecção pelo HIV no Brasil: uma epidemia multifacetada. Rev Soc Bras Med Trop. 2001;34(2):207-17.

15. Rasteiro JM, Oliveira JM. (In)segurança alimentar e nutricional em pessoas que vivem com HIV/AIDS atendidas no serviço especializado em moléstias infecto-contagiosas de Limeira, SP. Segur Aliment Nutr. 2014;21(1):337-46.

16. Charão APS, Batista MHRS, Ferreira LB. Food insecurity of HIV/AIDS patients at a unit of outpatient healthcare system in Brasilia, Federal District, Brazil. Rev Soc Bras Med Trop. 2012;45(6):751-3.

17. Tiyou A, Belachew T, Alemseged F, Biadgilign S. Food insecurity and associated factors among HIV-infected individuals receiving highly active antiretroviral therapy in Jimma zone Southwest Ethiopia. Nutr J. 2012;11(51):1-8.

18. Weiser SD, Yuan C, Guzman D, Frongillo EA, Riley ED, Bangsberg DR, et al. Food insecurity and HIV clinical outcomes in a longitudinal study of urban homeless and marginally housed HIV-infected individuals. AIDS. 2013;27(18):2953-8.

19. Anema A, Chan K, Chen Y, Weiser S, Montaner JSG, Hogg RS. Relationship between food insecurity and mortality among HIV-positive injection drug users receiving antiretroviral therapy in British Columbia, Canada. PLoS ONE. 2013;8(5):e61277.

20. Musumari PM, Wouters E, Kayembe PK, Nzita MK, Mbikayi SM, Suguimoto SP, et al. Food insecurity is associated with increased risk of non-adherence to antiretroviral therapy among HIV-infected adults in the Democratic Republic of Congo: a cross-sectional study. PLoS ONE. 2014;9(1):e85327.

21. Poblacion AP, Marín-León L, Segall-Corrêa AM, Silveira JA, Taddei JAAC. Insegurança alimentar em domicílios brasileiros com crianças menores de cinco anos. Cad Saude Publica. 2014;30(5):1067-78.

22. Galesi LF, Quesada KR, Oliveira MRM. Indicadores de segurança alimentar e nutricional. Rev Simbio-Logias. 2009;2(1):110.

23. Vasconcelos SML, Oliveira ACM, Torres NCP, Silva PMC, Santos TMP, Silva JVL, et al. Insegurança alimentar em domicílios de indivíduos portadores de hipertensão e/ou diabetes. Int J Cardiovasc Sci. 2015; 28(2)114-21.

\section{Endereço para correspondência:}

Lorena Nogueira Frota da Costa

Av. Antônio Justa, 3161

Bairro: Meireles

CEP 60165-090 - Fortaleza - CE - Brasil

E-mail: 1lorena.nutri@gmail.com 